\title{
ReMed: les médecins hospitaliers aussi
}

\author{
Le réseau d'assistance ReMed conseille et accompagne les médecins dans des moments \\ critiques - par exemple lorsque leur quotidien professionnel devient une trop grande \\ charge.
}

\section{Jürg Beutler}

Communication de la FMH

\begin{abstract}
Lors d'une prise de contact, ReMed réagit dans les 72 heures et examine la situation et les démarches individuelles possibles sans engagement de part et d'autre. ReMed est lié par le secret médical.
\end{abstract}

Avez-vous besoin de soutien? Un médecin de votre entourage nécessite-t-il une aide?

N'hésitez pas à contacter ReMed!

Ligne d'assistance téléphonique 24 h sur 24:

08000 73633,info@swiss-remed.ch, www.swiss-remed.ch

\section{Témoignage}

Un médecin hospitalier de 44 ans, marié et père de trois enfants, parle de la profonde dépression qu'il a traversée: a dépression s'est soudainement abattue sur moi, du service de traumatologie d'un assez grand (ravaillais tout seul, sans chef de clinique, avec le sens profond des responsabilités qui était le qui ne se laisse pratiquement pas exprimer par des mots. C'était un repli sur moi involontaire. J'avais perdu le contact oculaire avec mes patients, je voulais me débarrasser d'eux le plus rapidement possible, ce qui se reflétait dans les décisions thérapeutiques que je devais prendre. Au début, je mettais mon comportement sur le compte de mon manque de sommeil, mais j'ai rapidement constaté qu'il s'agissait d'autre chose de beaucoup plus grave. J'ai immédiatement appelé un collègue que je connaissais et j'ai pris rendez-vous chez lui. Cette rencontre n'a malheureusement pas été d'une grande aide pour moi, bien au contraire, j'ai eu l'impression de ne pas être considéré comme un patient.

Ma femme, par contre, a eu une réaction formidable. Elle a immédiatement réalisé ce qui se passait et a appelé mon ancien chef. Sans hésiter, il a envoyé une cheffe de clinique qui devait me remplacer et réduire ma charge de travail. Mon ancien chef a été un appui et un soutien important. Il m'a accompagné pendant cette crise, me rendait souvent visite, exactement ce dont j'avais besoin à ce moment-là. Je ne suis pas encore entièrement remis de ma dépression. Mais aujourd'hui je sais que je peux m'adresser à tout moment à ReMed et obtenir un soutien supplémentaire.

\section{Interview avec Jürg Bänninger}

Jürg Bänninger, vous êtes membre du Comité de direction de ReMed et vous êtes en contact direct avec les médecins concernés, quelles sont vos expériences?

Beaucoup de médecins attendent beaucoup trop longtemps avant de faire appel à notre aide, ils s'adressent à nous quand la situation leur paraît sans issue. C'est pour moi une mission très exigeante mais aussi passionnante: j'apporte mon aide aux médecins en détresse, recherche avec eux une voie de sortie et contribue en même temps à l'élaboration d'un réseau proche et approprié.

Vous regroupez pour ReMed les articles dont il est question ici. Pourquoi les médecins souhaitent-ils raconter leur histoire et la publier?

Jusqu'à présent, tous les collègues que j'ai sollicités pour une interview ont accepté. Les entretiens se déroulent dans une ambiance de confiance mutuelle. Et nous mettons un point d'honneur à ce que les témoignages soient publiés dans l'anonymat. Les interviewés ont naturellement la possibilité de relire les articles avant leur publication. Ces médecins racontent leur histoire parce qu'ils aimeraient éviter à d'autres ce qu'ils ont eux-mêmes vécu et parce qu'ils sont convaincus par l'idée de ReMed.

Que pouvez-vous conseiller aux médecins en détresse? Le médecin doit briser un tabou, celui de croire qu'il est obligé de s'aider par lui-même! Nous aussi, nous avons besoin d'un soutien professionnel! Parlez avec un ami de confiance ou un collègue dès les premiers signes avant-coureurs de difficultés. Si cela ne suffit pas: prenez contact avec ReMed le plus rapidement possible.

Avez-vous vécu une situation similaire et souhaiteriez-vous la raconter? Dans ce cas, n'hésitez pas à contacter ReMed. 